\title{
O adolescente e o diabetes: uma experiência de vida*
}

Teenagers with type 1 diabetes mellitus: life experience report

El adolescente y la diabetes: una experiencia de vida

\author{
Elaine Buchhom Cintra Damião', Vanessa Cristina Dias ${ }^{2}$, \\ Letícia Rosa de Oliveira Fabri ${ }^{3}$
}

\section{RESUMO}

Objetivo: Compreender como o adolescente com diabetes mellitus tipo I vivencia sua experiência de doença e como lida com esta situação no cotidiano. Métodos: O Interacionismo Simbólico foi utilizado como referencial teórico e a Teoria Fundamentada nos Dados como o referencial metodológico da pesquisa. Participaram do estudo 10 adolescentes com diagnóstico de diabetes mellitus tipo 1 há mais de um ano. Resultados: Foram identificados dois fenômenos explicativos da experiência: não sendo normal ter diabetes e sendo normal ter diabetes . Conclusão: Os dois fenômenos não são isolados ou excludentes para o mesmo adolescente, parecendo haver períodos ou fases em que os adolescentes identificam-se e vivenciam ora um fenômeno ora outro, com maior ou menor intensidade.

Descritores: Adolescente; Diabetes mellitus tipo 1; Enfermagem pediátrica.

\begin{abstract}
Purpose: To understand the life experience of teenagers with type 1 diabetes mellitus and how they cope with the disease in daily life. Methods: The symbolic interactionism was the theoretical perspective guiding the study. Grounded theory served as the method for using the empirical data. Participants consisted of 10 teenagers with type 1 diabetes mellitus over a year. Results: Two phenomena explaining their life experience emerged: "Regarding having diabetes as being normal" and "regarding having diabetes as being abnormal." Conclusion: The two phenomena were not isolated or excluded from the life experience of the same teenager; there were phases in which the teenagers experienced one of the phenomena and vice-versa with greater or less intensity.
\end{abstract}

Key Words: Teenagers; Type 1Diabetes Mellitus; Pediatric Emergency.

\section{RESUMEN}

Objetivo: Comprender como el adolescente con diabetes mellitus tipo I experimenta su enfermedad y como lidia con esta situación en lo cotidiano. Métodos: El Interaccionismo Simbólico fue utilizado como marco teórico y la Teoría Fundamentada en los Datos como el marco metodológico de la investigación. Participaron del estudio 10 adolescentes que tenían diagnóstico de diabetes mellitus tipo 1 hace más de un año. Resultados: Fueron identificados dos fenómenos que explicaban la experiencia: no es normal tener diabetes y es normal tener diabetes. Conclusión: Los dos fenómenos no están aislados o se excluyen mutuamente para el mismo adolescente, pareciendo haber períodos o fases en que los adolescentes se identifican y viven una vez un fenómeno y otra vez el otro, con mayor o menor intensidad. Palabras clave: Adolescente; Diabetes mellitus tipo 1; Enfermería pediátrica.

\footnotetext{
* Estudo vinculado ao projeto de pesquisa "Viver com diabetes mellitus tipo 1: a experiência do adolescente e de sua família". Projeto de pesquisa financiado pelo CNPq - Edital Universal sob n 478780/2006-0.

1 Doutora em Enfermagem. Professora do Departamento de Enfermagem Materno-Infantil e Psiquiátrica da Escola de Enfermagem da Universidade de São Paulo - São Paulo (SP), Brasil.

2.3 Acadêmicas do 80. Semestre do Curso de Graduação em Enfermagem da Escola de Enfermagem da Universidade de São Paulo - São Paulo (SP), Brasil. Bolsistas de Iniciação Científica pela Fundação de Amparo à Pesquisa do Estado de São Paulo.
} 


\section{INTRODUÇÃO}

A adolescência é uma fase difícil para o ser humano, porque além de ser um período de transição entre a infância e a idade adulta, é também um momento de muitas mudanças que ocorrem nos aspectos físico, cognitivo, emocional e social. O adolescente com doença crônica, além disso, também precisa enfrentar as situações causadas pela doença ao seu cotidiano, destacando-se o diabetes mellitus como a endocrinopatia mais comum da infância ${ }^{(1-2)}$.

Segundo a International Diabetes Federation 70.000 crianças desenvolvem diabetes mellitus (DM) tipo 1 a cada ano, e cerca de 200 crianças desenvolvem a doença a cada $\operatorname{dia}^{(3)}$. No Brasil, existem atualmente cerca de 5 milhões de diabéticos, dos quais cerca de 300 mil são menores de 15 anos de idade $^{(4)}$. O diabetes mellitus tipo I, que acomete em geral crianças e adolescentes, é uma doença que pode ser administrada através de quatro pilares básicos: insulinoterapia, dietoterapia, monitorização do controle glicêmico e exercício físico ${ }^{(5)}$.

$\mathrm{O}$ adolescente sente-se limitado pela doença em si e pelas restrições por ela impostas, fazendo com que ele sofra ao perceber-se diferente do grupo e ao ser tratado diferentemente pelas pessoas ${ }^{(6-8)}$. A família, no entanto, vive preocupada e ansiosa em relação ao tratamento do filho, temendo as complicações imediatas e tardias quando o adolescente não segue o tratamento corretamente ${ }^{(9)}$.

Vários estudos têm sido realizados, destacando-se o Diabetes Control and Complications Trial ${ }^{(10)}$, que revolucionou a terapêutica do $\mathrm{DM}$ ao comprovar que o tratamento intensivo do DM produz melhor controle glicêmico, redução do risco das complicações e melhor qualidade de vida. A literatura sobre DM é prolífica, porém a maioria dos estudos enfoca a fisiopatologia ou o tratamento da doença; poucos abordam os aspectos psicossociais. Estes apresentam questões como qualidade de vida e auto-imagem, bem como as dificuldades encontradas pelo adolescente e pela família no manejo do diabetes, como comunicação e conflito com os pais e a participação dos pais no tratamento do $\mathrm{DM}^{(11-17)}$. Entretanto, somente algumas pesquisas buscam compreender a experiência do adolescente ao conviver com o $\mathrm{DM}^{(18-22)}$, inclusive em nosso meio ${ }^{(23-25)}$, considerando a magnitude e as implicações que a doença acarreta ao adolescente. Sabemos que conhecer como o adolescente pensa e se comporta em relação à situação de doença é essencial para a proposição de intervenções que venham ao encontro de suas reais necessidades. Desse modo, este estudo teve como objetivo compreender como o adolescente com diabetes mellitus tipo I vivencia sua experiência de doença e como lida com esta situação no cotidiano.

\section{MÉTODOS}

Adotou-se o Interacionismo Simbólico (IS) como referencial teórico e a Teoria Fundamentada nos Dados (TFD) como o referencial metodológico da pesquisa.

O IS é uma teoria sobre o comportamento humano e se fundamenta no pressuposto de que a experiência humana é mediada pela interpretação ${ }^{(26)}$. Nessa perspectiva, considerou-se o adolescente um expert para descrever sua situação de doença e, através do seu relato, pode-se compreender qual o significado que o adolescente com DM tipo 1 atribui à experiência de conviver com a situação de doença diariamente.

A TFD, desenvolvida pelos sociólogos americanos Barney Glaser e Anselm Strauss, é um método de pesquisa qualitativa, destinada a descobrir novos conceitos e teorias por meio de dados da realidade, ao invés de testar dados já existentes. $\mathrm{O}$ processo de coleta de dados requer que o pesquisador colete o dado, proceda a sua codificação e análise de modo concomitante, o que permite ao pesquisador decidir qual tipo de dado deverá ser coletado a seguir, e onde será possível encontrá-lo. A análise dos dados é realizada através do método de comparação constante, possibilitando a emersão das categorias e subcategorias, até o desvelar de uma teoria explicativa da experiência que está sendo investigada ${ }^{(27)}$.

Fizeram parte do estudo dez adolescentes (cinco meninas e cinco meninos) com idade de 12 a 18 anos com diagnóstico de diabetes mellitus tipo 1 há mais de um ano. O período de um ano após o diagnóstico foi estabelecido a fim de que o adolescente tivesse tempo de se recuperar do impacto do diagnóstico, além de permitir que tivesse vivenciado a experiência de doença. A escolha dos participantes ocorreu de modo a privilegiar a variedade de experiências vivenciadas pelo adolescente. Desse modo, tivemos adolescentes de ambos os sexos, com diferentes idades, isto é, no início, meio e final do período da adolescência e procedentes de diferentes instituições hospitalares e associações como a Associação de Diabetes Juvenil e a Casa do Caminho em Jundiaí, São Paulo.

Após a aprovação pelo Comitê de Ética em Pesquisa da Escola de Enfermagem da Universidade de São Paulo, os adolescentes elegíveis foram convidados a participar do estudo, tendo sido explicado e solicitado à mãe ou responsável pelo adolescente a autorização para que o mesmo participasse da pesquisa. Depois de receberem todos os esclarecimentos, solicitou-se que o adolescente assinasse o Termo de Consentimento para Participar da Pesquisa Científica e seu responsável assinasse o Termo de Consentimento Livre e Esclarecido.

Os dados foram coletados no período de março a novembro de 2007, através de entrevistas semi-estruturadas, áudiogravadas em local privativo, com duração de 30 minutos, em média, e teve como questão norteadora: "Conte-me como é, para você, ter uma doença como o diabetes". Novas questões foram acrescidas a fim de se obter esclarecimentos sobre os dados e fundamentar a experiência do adolescente em ter uma doença crônica como o DM tipo 1.

O processamento das falas dos sujeitos iniciou-se com a transcrição fiel das fitas gravadas. Foram, então, extraídos os códigos, que são as unidades básicas de análise. Durante a codificação aberta, os dados foram "quebrados" em pequenas frases, examinados, comparados por similaridades e diferenças, sendo também feitas perguntas aos códigos sobre o fenômeno contido nos dados. Estes dois procedimentos 
analíticos são básicos para a codificação dos dados: “fazer comparações" e "fazer perguntas" a cada incidente, evento e a outras facetas do fenômeno. Os dados codificados foram agrupados por similaridade de fenômeno. Esse processo de agrupar conceitos relativos ao mesmo fenômeno é chamado de categorização. Uma categoria está saturada quando não é possível acrescentar novos dados ou quando não mais emergem novos aspectos representativos do fenômeno ${ }^{(28)}$.

Essa metodologia, por ser uma forma em que os dados são manejados pelo pesquisador, e sendo uma construção constante, permite que os resultados sejam relatados, sendo possível a apresentação da teoria, do fenômeno ou de uma categoria $^{(28)}$. No presente estudo, de acordo com o objetivo proposto, a análise dos dados resultou em dois fenômenos e categorias explicativas da experiência do adolescente com DM tipo 1.

\section{RESULTADOS}

A situação de doença crônica é vivenciada pelo adolescente com DM tipo 1 de forma multifacetada. Os fenômenos identificados a partir dos dados, "não sendo normal ter diabetes" e "sendo normal ter diabetes" devem ser compreendidos interacionalmente, pois representam o significado que o adolescente atribui a sua experiência de doença, a partir da sua própria perspectiva. Entretanto, os fenômenos não devem ser compreendidos como estágios lineares, seqüenciais e obrigatórios da vivência de todos os adolescentes com diabetes. Nesse sentido, o adolescente pode identificar-se e vivenciar ora um fenômeno ora outro, com maior ou menor intensidade. Uma outra situação possível é o adolescente experienciar apenas um dos fenômenos, como podemos depreender dos relatos dos adolescentes participantes deste estudo. Parte dos adolescentes refere que, na época do diagnóstico, o diabetes não era normal em sua vida, mas que com o passar do tempo, aprendeu a lidar com a situação de doença, tornando o diabetes parte de sua rotina, sendo normal a convivência com sua doença. Outros adolescentes, no entanto, parecem estar presos a suas dificuldades em lidar com o diabetes, sentindo que para eles não é normal ter diabetes, sendo um peso difícil de ser carregado, sobrecarregando-os de sofrimento. A seguir, apresentamos os fenômenos e as respectivas categorias descritivas (Figura 1).

\section{Não sendo normal ter diabetes}

Este fenômeno é caracterizado pelo sofrimento e insegurança que o adolescente experiencia ao ter de conviver com o DM tipo 1. A maioria dos adolescentes experimenta situações problemáticas no dia-a-dia com seus pares, professores e familiares, e mesmo com o próprio tratamento. Muitas vezes parece que o fardo é pesado demais para ser carregado.

\section{Não aceitando a doença}

Esta categoria retrata a dificuldade que o adolescente tem de reconhecer o diabetes como uma doença incurável, com a qual ele deve conviver. A situação de doença nao faz sentido para o adolescente, mesmo quando ele é confrontado com a realidade do tratamento, seja realizando a monitorização glicêmica ou as injeções diárias de insulina.

"Antes, eu não pensava que tinha diabetes, en não aceitava a doença..." (S10)

\section{Tendo muitos problemas ao lidar com o diabetes}

Enquanto a categoria anterior descreve os aspectos relativos ao ser adolescente, esta categoria retrata os problemas que o adolescente enfrenta ao lidar com a parte prática do tratamento do diabetes. A dieta é vista como um dos mais difíceis aspectos da vivência do diabetes. $\mathrm{O}$ adolescente sentese chateado por "passar vontade", por não poder comer as mesmas coisas que os amigos ou ainda por não poder comer o que quiser, quando e como quiser.

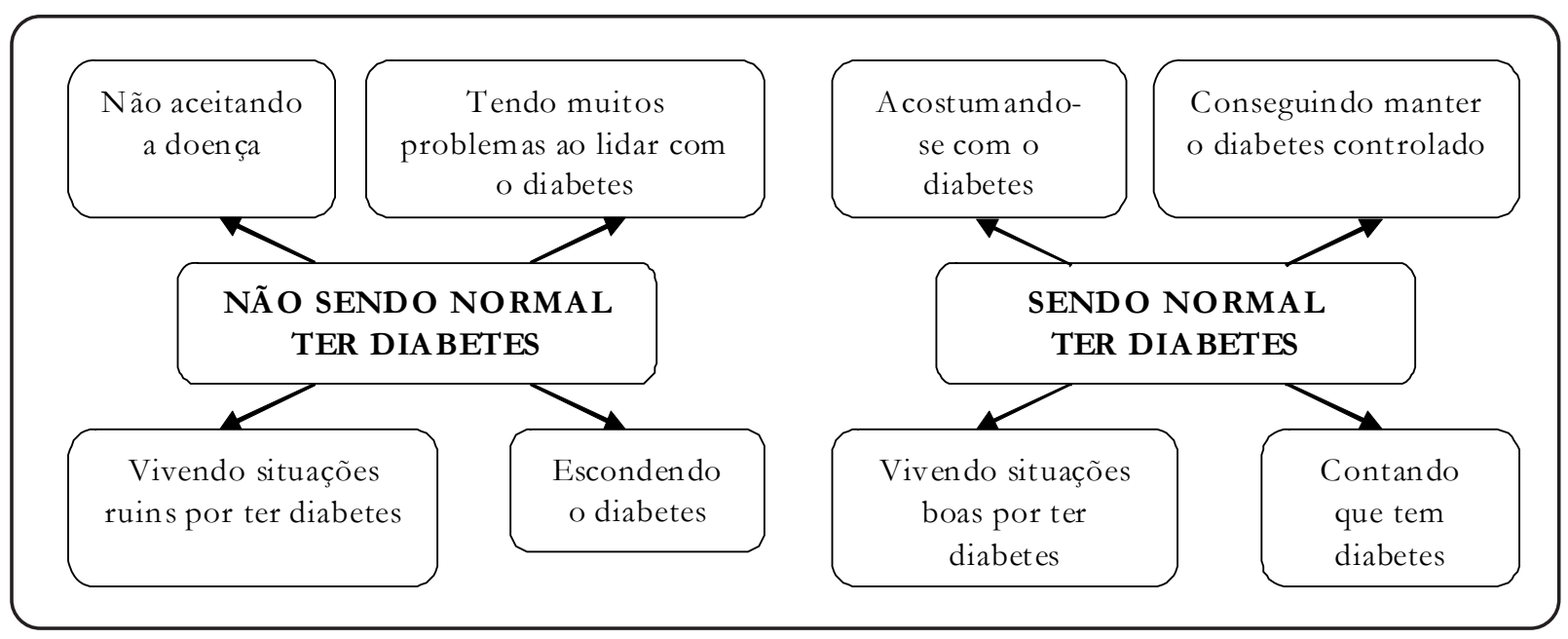

Figura 1. Categorias descritivas dos fenômenos "não sendo normal ter diabetes" e "sendo normal ter diabetes". 
O aumento do número de injeções de insulina é motivo de grande sofrimento para o adolescente, apesar de ser visto como natural pela equipe de saúde, pois a criança ou adolescente já vem recebendo uma ou mais doses de insulina. Outro aspecto refere-se à auto-aplicação de insulina, ao relatar que algumas vezes suprime doses de insulina, ou aplica somente a insulina NPH, não fazendo as correções necessárias com a insulina de ação rápida ou ultra-rápida.

As complicações agudas do diabetes, hipoglicemias e hiperglicemias, são ocorrências que podem sobrevir no diaa-dia do adolescente. $\mathrm{O}$ adolescente, em geral, preocupa-se mais com a hipoglicemia, porque esta, quando severa, pode acarretar desmaios, convulsões ou comportamentos inadequados, trazendo muito constrangimento para o adolescente.

"Teve uma vez que en estava na editora e o sol estava muito forte naquele dia e eu tive uma hipoglicemia. E eu acabei desmaiando..." (S3)

\section{Vivendo situações ruins por ter diabetes}

$\mathrm{O}$ adolescente relata que vive muitas situações desagradáveis em decorrência do diabetes. Sofre e sente-se impotente diante das ocorrências a que é exposto, reforçando a condição de que não é normal ter diabetes e tornando ainda mais difícil a adaptação à situação de doença. Desse modo, algumas vezes o adolescente é colocado em situações constrangedoras por ter diabetes. Mesmo que o motivo seja elogiar o adolescente por ser capaz de lidar com a doença ou servir de exemplo para os pares que não têm nenhum tipo de doença, o adolescente detesta ser apontado ou destacado por ter diabetes.

As interações sociais são bastante importantes para o adolescente, principalmente com seus pares. No entanto, os colegas podem tornar a vida do adolescente bastante difícil ao pressioná-lo a comer ou beber alimentos não apropriados a sua dieta, como em festas onde o grupo estimula o consumo de alimentos e bebidas não diet, tornando as escolhas do adolescente difíceis. Muitas vezes, ele não consegue ser firme o suficiente para manter sua posição e consumir os alimentos apropriados a sua condição.

"Quando eu vou a festas, às vezes não tem refrigerante diet e os meus amigos ficam insistindo para que eu tome refrigerante normal. Eu não quero fazer isso, mas eles insistem tanto, que eu não consigo dizer não. É complicado..." (S4)

\section{Escondendo o diabetes}

A maioria das crianças e adolescentes quando diagnosticados com diabetes não se sente à vontade para dizer que tem diabetes. Nesse caso, o adolescente passa a esconder sua situação de doença. Esconder o diabetes é um processo que toma muita energia do adolescente porque tem que pensar no que dizer, tem que, literalmente, se esconder em banheiros para realizar a monitorização glicêmica e as injeções de insulina, quando necessário, inventar desculpas pelos horários que tem de cumprir em relação à alimentação, por exemplo. Outro comportamento que o adolescente pode apresentar é ignorar o tratamento enquanto está com colegas e amigos, fingindo até para si mesmo, que não tem necessidade de realizá-lo.

$\mathrm{O}$ adolescente refere que esconde o fato de ter diabetes por duas razões: vergonha e o medo de ser rejeitado pelos amigos quando souberem que ele tem diabetes. Vergonha e medo da rejeição são dois fortes sentimentos que podem motivar comportamentos, mesmo inadequados.

"...antigamente eu tinha muitos problemas, porque eu escondia de muita gente, não falava pra ninguém na escola..." (S7)

"Quando eu era bem pequeno, com uns 5, 6 anos de idade, eu tinha um medo de falar, uma vergonha de falar para os outros que en tinha diabetes." (S8)

\section{Sendo normal ter diabetes}

Este fenômeno retrata a convivência e aceitação do adolescente em relação a sua condição de ter uma doença como o diabetes com confiança, responsabilidade e apoio dos amigos, familiares e profissionais, incorporando as ações relacionadas ao tratamento e a sua rotina de vida.

\section{Acostumando-se com o diabetes}

Geralmente o adolescente, quando recebe o diagnóstico do diabetes, inicia um processo de buscar informações para conhecer melhor a doença e, desta maneira, adaptar-se a sua nova condição. Esta procura permite que ele entenda quais são as consequências que a doença traz ao seu organismo e, também, se conscientize e aprenda a lidar com o diabetes. Compreender o que é uma doença como o diabetes permite que ele encare de maneira diferente o tratamento, e tenha mais segurança quanto à auto-aplicação de insulina, monitorização da glicemia, dieta equilibrada e a prática regular de exercícios. $\mathrm{O}$ adolescente nota que, depois de algum tempo, é mais fácil lidar com a doença, acostumando-se com sua nova condição.

"...depois vai acostumando. A gente no começo liga muito para o quê os outros falam, depois a gente descobre outras pessoas, vai conbecendo melhor essa doença."(S2)

\section{Conseguindo manter o diabetes controlado}

$\mathrm{O}$ adolescente, com o intuito de manter o diabetes controlado, busca estratégias que facilitem o manejo da doença. Alguns deles relatam que hoje é mais fácil controlar o diabetes do que antigamente. No início da doença, o adolescente refere não ter a dimensão exata do que significa ter diabetes e quais serão suas consequências. No entanto, conforme o tempo passa, ele reconhece que para ficar bem necessita seguir o tratamento prescrito, conscientizando-se sobre a sua condição, a de ser um adolescente com DM tipo 1. A conscientização do adolescente é um momento muito importante, porque ele passa a considerar o diabetes como algo não desejado, porém que não é incompatível com seu estilo de vida, conseguindo manter-se controlado. 
"Eu tenho a responsabilidade agora de me cuidar sozinho, estudo, trabalho, faço aplicações fora de casa, vou passear, viajar por semanas, isso me ajudou bastante sim.” (S8)

\section{Vivendo situações boas por ter diabetes}

A experiência do adolescente com DM tipo 1 revela que este também vivencia situações boas, até mesmo prazerosas por ter diabetes. Muitos adolescentes eram obesos ou com tendência à obesidade anteriormente ao diagnóstico, porém como neste momento emagrece muito devido à descompensação metabólica e conseqüente cetoacidose, a perda de peso torna-se um ganho secundário que é valorizado pelo adolescente.

"Mas uma situação boa foi que eu emagreci, eu emagreci uns 7 a 8 quilos, então eu acho que pra mim foi uma parte boa, ter emagrecido..." (S2)

Muitos adolescentes relatam que quando descobriram o diabetes foram encaminhados a associações para pessoas com diabetes e seus familiares, a fim de terem auxílio nos insumos e acesso a informações, bem como apoio de profissionais da área de saúde e dos pares. A convivência com pessoas que tem a mesma condição, no caso o diabetes, faz com eles sintam-se à vontade para compartilhar seus sucessos e insucessos no manejo do diabetes, fazendo ainda novos amigos. Eles vêem esta situação como bastante positiva, porque eles conhecem muitas pessoas nas associações, passando a perceber que eles não são os únicos a ter que lidar com o diabetes.

"O lado bom foi que eu conheci muita gente na ADJ. Se eu não tivesse ido, não teria ido pro acampamento, não teria participado, eu não estaria aqui hoje. Eu não conbecia nada.” (ST)

\section{Contando que tem diabestes}

Uma característica própria da adolescência refere-se à necessidade de autonomia e de ser aceito pelo grupo, por isso nem sempre é fácil para o adolescente revelar com naturalidade que tem diabetes. No entanto, a partir do momento em que ele mesmo encara com naturalidade e normalidade o ser diabético, percebe que não tem mais problemas, que não se sente envergonhado em contar aos amigos e parentes que tem diabetes.

"...eu voltei do acampamento com essa idéia, porque se eles gostam de mim pensando assim: que eu sou uma pessoa que não tem nada, não sabendo que eu tenho diabetes, eles vão gostar de mim do mesmo jeito, sabendo que eu tenho diabetes, que isso não é uma coisa, um bicho de sete cabeças, não é? É uma coisa normal, é só controlar." (ST)

\section{DISCUSSÃO}

A necessidade de normalização está presente na maioria das famílias e das pessoas que convivem com uma doença crônica. $\mathrm{O}$ conceito de normalização tem sido discutido na literatura científica desde o início da década de 1980, principalmente relacionado ao papel que a família exerce no cotidiano da criança com doença crônica. Normalização é definida como um processo ativo de acomodação ativo das necessidades físicas e emocionais da criança e do adolescente cronicamente doente, sem que a doença ou a criança doente torne-se o centro do sistema familiar ${ }^{(29-34)}$.

Este conceito não tem sido utilizado em relação ao indivíduo, estando mais associado às tarefas que a família deve executar para adaptar-se bem à situação de doença do filho. Entretanto, acreditamos que este conceito tem grande aplicabilidade na experiência do adolescente com DM. Ser normal, não ser diferente de seus pares faz parte dos anseios de qualquer adolescente. No caso do adolescente com DM, parte deles acredita na normalidade de sua vida, na qual a situação de ter diabetes está inserida. Para eles, a normalidade não significa a ausência ou a negação da doença, mas que a doença faz parte da normalidade de vida que ele pode ter, sem que aconteça sofrimento ou estresse excessivo.

O fato do adolescente encarar sua vida e doença com normalidade ajuda-os a manter um bom controle do diabetes. Eles então assumem seu tratamento, responsabilizando-se por ter as atitudes corretas frente à insulinoterapia, monitorização glicêmica, dietoterapia e a prática de exercícios, sendo capazes de fazer as correções necessárias nos casos de hiper ou hipoglicemia. Ele compreende que a responsabilidade adquirida em relação ao tratamento traz recompensas, e que o envolvimento com outras pessoas, seja individualmente ou em grupos, reforça sua autonomia e independência.

Entretanto, muitos adolescentes continuam desejando ser normais como eram antes de ter a doença, mesmo quando bem orientados a respeito da doença e sabendo que o diabetes é uma doença crônica. Este comportamento pode indicar que o adolescente esteja tendo dificuldades em lidar com a situação de doença.

Lidar com as demandas da doença e de seu crescimento e desenvolvimento não é uma tarefa fácil para o adolescente, podendo resultar em escolhas inadequadas ao tratamento do diabetes, em detrimento de sua saúde, muitas vezes por não querer ser visto como uma pessoa doente por seus pares.

$\mathrm{O}$ adolescente percebe que o tratamento pode interferir de maneira positiva ou negativa em sua necessidade de autonomia e independência, que são características marcantes desta fase do desenvolvimento. Este é o período que, em geral, o adolescente apresenta maior número de comportamentos inapropriados em relação ao tratamento do diabetes, por ter medo de sentir-se diferente de seus pares e/ou de não ser mais aceito pelo grupo. Em alguns casos, o adolescente pode negligenciar seu tratamento, deixando de seguir as orientações quanto à dieta, falsificando os resultados do monitoramento da glicemia capilar, ou mesmo suprimindo uma ou outra dose de insulina, havendo, como consequência, alterações maiores da glicemia e por fim a descompensação e cetoacidose diabética ${ }^{(35-37)}$.

O apoio dos pares ou a falta dele é um fato também descrito pela literatura. A relação com os pares e amigos exerce um papel importante no desenvolvimento emocional do 
adolescente. Sendo assim, muitas vezes relega seu tratamento, por sentir vergonha ou pela insistência dos amigos ao consumo de bebidas e alimentos não recomendados pelo tratamento. Desse modo, a influência dos pares tanto pode ser positiva, ao apoiar o adolescente em seu tratamento, diminuindo o impacto dos estressores, quanto negativa, ao estimular o adolescente a quebrar a rotina e orientações fornecidas pela equipe de saúde ${ }^{(38-42)}$.

\section{CONSIDERAÇÕES FINAIS}

Este estudo vem agregar novos conhecimentos à temática, e em especial a uma área ainda praticamente inexplorada, ao desvelar aspectos da experiência de doença do adolescente com DM tipo1.

Os dados apresentados pelos adolescentes participantes permitem-nos destacar duas questões: a primeira diz respeito ao fato de quanto a normalização da vida do adolescente é importante para ele; e a segunda, refere-se a importância positiva que os pares, as associações e grupos de suporte exercem sobre a experiência de doença do adolescente, auxiliando-o a lidar melhor com seu diabetes no cotidiano.

Nesse sentido, as enfermeiras devem estar atentas para

\section{REFERÊNCIAS}

1. Leal MM, Saito MI. Singularidades do desenvolvimento do adolescente: a síndrome da adolescência normal. In: Marcondes E, Vaz FAC, Ramos JLA, Okay Y. Pediatria básica. 9a. ed. São Paulo: Sarvier; 2002. p. 666-9.

2. Muscari ME. Coping with chronic illness. Nursing knowledge and compassion can empower ill or disabled teens. Am J Nurs. 1998;98(9):20-2.

3. International Diabetes Federation. Today's children to bear brunt of diabetes epidemic [Internet]. USA: International Diabetes Federation; 2006 [cited 2007 Jul 4]. Available from: http: / / www.idf.org $/$ node $/ 1350$ ? unode=F0F11E 53-84FB-4D49-8984-86684BC05920

4. Ministério da Saúde (BR). Organização Pan-Americana da Saúde. Avaliação do plano de reorganização da atenção à hipertensão arterial e ao diabetes mellitus no Brasil. Brasília: Ministério da Saúde; 2004.

5. Grossi SAA, Cianciarullo TI, Della Manna T. Avaliação de dois esquemas de monitorização domiciliar em pacientes com diabetes mellitus do tipo 1. Rev Esc Enferm USP. 2002;36(4):317-23

6. Marcelino DB, Carvalho MDB. Reflexões sobre o diabetes tipo 1 e sua relação com o emocional. Psicol Reflex Crit. 2005;18(1):72-7.

7. Woodgate RL. Adolescents' perspectives of chronic illness: "It's hard". J Pediatr Nurs. 1998;13(4):210-23.

8. Tracy JP. Growing up with chronic illness: the experience of growing up with cystic fibrosis. Holist Nurs Pract. 1997;12(1):27-35.

9. Silva LF, Guedes MVC, Moreira RP, Souza ACC. Doença crônica: o enfrentamento pela família. Acta Paul Enferm. 2002;15(1):40-7. compreender a experiência do adolescente e propor intervenções que o ajudem e a sua família a normalizar a sua rotina de vida, para que cada vez mais o adolescente com DM acredite que ele pode ser normal e ter diabetes, ao vivenciar uma vida plena.

O enfermeiro, além disso, deve estar capacitado a promover intervenções levando em consideração que, apesar dos adolescentes não quererem ser diferentes das outras pessoas e em especial de seus pares, eles necessitam de um cuidado diferenciado e compartilhado. Deve-se, também estimular e reconhecer os esforços dos adolescentes que acreditam ser normal ter diabetes e mostrar-lhes que seu empenho é valorizado pela equipe de saúde. Aos adolescentes com dificuldades para lidar com a situação de doença, devemos auxiliá-los a recuperar a auto-estima, confiança, bem como a autonomia, diante da situação de doença, tendo uma vida mais produtiva e com menos sofrimento.

Apesar de não podermos generalizar os resultados obtidos, a pesquisa nos permite ter a compreensão clara da natureza íntima deste grupo de adolescentes em relação a sua experiência de doença, podendo ser utilizada como abordagem inicial no atendimento da enfermeira ao adoles-cente com DM tipo 1.

10. The effect of intensive treatment of diabetes on the development and progression of long-term complications in insulin-dependent diabetes mellitus. The Diabetes Control and Complications Trial Research Group. N Engl J Med. 1993;329(14):977-86.

11. Faukner MS. Quality of life for adolescents with type 1 diabetes: parental and youth perspectives. Pediatr Nurs. 2003;29(5):362-8.

12. Erkolahti RK, Ilonen T, Saarijärvi S. Self-image of adolescents with diabetes mellitus type 1 and rheumatoid arthritis. Nord J Psychiatry. 2003;57(4):309-12.

13. Hanna KM, Juarez B, Lenss SS, Guthrie D. Parentadolescent communication and support for diabetes management as reported by adolescents with type 1 diabetes. Issues Compr Pediatr Nurs. 2003;26(3):145-58.

14. Wagner JA, Abbott G, Lett S. Age related differences in individual quality of life domains in youth with type 1 diabetes. Health Qual Life Outcomes. 2004;2:54.

15. Viikinsalo MK, Crawford DM, Kimbrel H, Long AE, Dashiff C. Conflicts between young adolescents with type I diabetes and their parents. J Spec Pediatr Nurs. 2005;10(2):69-79; discussion79-80.

16. Beveridge RM, A Berg C, J Wiebe D, L Palmer D. Mother and adolescent representations of illness ownership and stressful events surrounding diabetes. J Pediatr Psychol. 2006;31(8):818-27.

17. Helgeson VS, Novak SA. Illness centrality and well-being among male and female early adolescents with diabetes. J Pediatr Psychol. 2007;32(3):260-72.

18. Amer KS. Children's views of their adaptation to type 1 diabetes mellitus. Pediatr Nurs. 2008;34(4):281-8. 
19. Huus K, Enskär K. Adolescents' experience of living with diabetes. Paediatr Nurs. 2007;19(3): 29-31.

20. Herrman JW. Children's and young adolescents' voices: perceptions of the costs and rewards of diabetes and its treatment. J Pediatr Nurs. 2006;21(3):211-21.

21. Buchbinder MH, Detzer MJ, Welsch RL, Christiano AS, Patashnick JL, Rich M. Assessing adolescents with insulin-dependent diabetes mellitus: a multiple perspective pilot study using visual illness narratives and interviews. J Adolesc Health. 2005;36(1):71.e9-13.

22. Dickinson JK, O'Reilly MM. The lived experience of adolescent females with type 1 diabetes. Diabetes Educ. 2004;30(1):99-107.

23. Zanetti ML, Otero LM, Peres DS, Santos MA, Guimarães FPM, Freitas MCF. Progress of the patients with diabetes mellitus who were managed with the staged diabetes management framework. Acta Paul Enferm. 2007;20(3): 338-44.

24. Sampaio FAA, Melo RP, Rolim ILTP, Siqueira RC, Ximenes LB, Lopes MVO. Evaluation of the health promotion behavior in patients with diabetes mellitus. Acta Paul Enferm. 2008;21(1):84-8.

25. Novato TS, Grossi SAA, Kimura M. Cultural adaptation and validation of the "Diabetes Quality of Life for Youths" measure of Ingersoll and Marrero into Brazilian culture. Rev Latinoam Enferm. 2008;16(2): 224-30.

26. Charon JM. Symbolic interactionism: an introduction, an interpretation, an integration. 5th ed. New Jersey: Simon \& Schuster; 1995.

27. Glaser BG, Strauss AL. The discovery of grounded theory: strategies for qualitative research. Hawthorne: Aldine de Gruyter; 1967.

28. Strauss AL, Corbin J. Basics of qualitative research: grounded theory procedures and techniques. Newbury Park, Calif.: Sage Publications; c1990.

29. Knafl KA, Deatrick JA. How families manage chronic conditions: an analysis of the concept of normalization. Res Nurs Health. 1986;9(3):215-22.

30. Deatrick JA, Knafl KA, Walsh M. The process of a parenting a child with a disability: normalization through accommodations. J Adv Nurs. 1988;13(1):15-21.

31. Bossert E, Holaday B, Harkins A, Turner-Henson A. Strategies of normalization used by parents of chronically ill school age children. J Child Adolesc Psychiatr Ment Health Nurs. 1990;3(2):57-61.

32. Deatrick JA, Knafl KA, Murphy-Moore C. Clarifying the concept of normalization. Image J Nurs Sch. 1999;31(3):209-14.

33. Knafl KA, Deatrick JA. The challenge of normalization for families of children with chronic conditions. Pediatr Nurs. 2002;28:49-56.

34. Silverstein J, Klingensmith G, Copeland K, Plotnick L, Kaufman F, Laffel L, et al. American Diabetes Association. Care of children and adolescents with type 1 diabetes: a statement of the American Diabetes Association. Diabetes Care. 2005;28(1):186-212.

35. Weissberg-Benchell J, Glasgow AM, Tynan WD, Wirtz P, Turek J, Ward J. Adolescent diabetes management and mismanagement. Diabetes Care. 1995;18(1):77-82.

36. Damião EBC, Pinto CMM. Being transformed by illness: adolescents' diabetes experience. Rev Latinoam Enferm. 2007;15(4):568-74.

37. Pinto CMM, Damião EBC. Lidando com o diabetes: a experiência do adolescente no cotidiano. Rev Paul Enferm. 2007;26(3).

38. Greco P, Pendley JS, McDonell K, Reeves G. A peer group intervention for adolescents with type 1 diabetes and their best friends. J Pediatr Psychol. 2001;26(8):485-90.

39. La Greca AM, Bearman KJ, Moore H. Peer relations of youth with pediatric conditions and health risks: promoting social support and healthy lifestyles. J Dev Behav Pediatr. 2002;23(4):271-80.

40. Hains AA, Berlin KS, Davies WH, Parton EA, Alemzadeh R. Attributions of adolescents with type 1 diabetes in social situations: relationship with expected adherence, diabetes stress, and metabolic control. Diabetes Care. 2006;29(4):818-22.

41. Hains AA, Berlin KS, Davies WH, Smothers MK, Sato AF, Alemzadeh R. Attributions of adolescents with type 1 diabetes related to performing diabetes care around friends and peers: the moderating role of friend support. J Pediatr Psychol. 2007;32(5):561-70.

42. Hains AA, Berlin KS, Davies WH, Sato AF, Smothers MK, Clifford LC, Alemzadeh R. Attributions of teacher reactions to diabetes self-care behaviors. J Pediatr Psychol. 2009;34(1):97-107. 